\title{
Interest of a Broad Vessel Carrier of the Sural Neuro-Cutaneous Flap for the Covering of Post-Traumatic Skin Defects of the Ankle and Foot
}

\author{
Ababou $\mathrm{K}^{1 *}$, Ribag $\mathrm{Y}^{1}$, Fouadi $\mathrm{F}^{1}$, Khales $\mathrm{A}^{1}$, Achbouk $\mathrm{H}^{1}$, Ouardi $\mathrm{A}^{1}$, Badaoui $\mathrm{Z}^{1}$, Moqfi $\mathrm{I}^{1}$, Nassim Sabah $\mathrm{T}^{2}$, EL
} Khatib $\mathrm{M} \mathrm{K}^{3}$, Siah $\mathrm{S}^{1}$

${ }_{1}^{1}$ Service de Chirurgie Plastique, Réparatrice et des Brûlés, Hôpital Militaire d'Instruction Mohamed V, Rabat, Maroc
${ }^{2}$ Service de Chirurgie Plastique, Hôpital Militaire, Agadir Morocco
${ }^{3}$ Service de Chirurgie maxillo-faciale et stomatologie, Hôpital Militaire d'Instruction Mohamed V, Rabat, Maroc

DOI: $10.36347 /$ sjmcr.2020.v08i01.008

| Received: 01.01.2020 | Accepted: 10.01.2020 | Published: 14.01.2020

*Corresponding author: Ababou Karim

\section{Abstract}

Original Research Article

Introduction: The reconstruction of defects in the foot and ankle is a real challenge for plastic surgeons. Material and methods: A retrospective study of a series made up of 18 patients who benefited, in the plastic surgery department of the Mohamed V Military Hospital in Rabat, from January 2015 to December 31, 2018, of a reconstruction of ankle skin substance loss and of the neck of the foot by a sural neurocutaneous flap with distal pedicle, using a fascio-fatty strip containing the pedicle more than $3 \mathrm{~cm}$. while specifying the inclusion and exclusion criteria. Results: We noticed, a male predominance. The average age is 41.6 years old. The coverage interested the internal malleolus in ten cases, the external malleolus in seven cases, and the instep for 3 patients. Ten out of eighteen patients benefited from an intervention in two surgical times, covering the skin defect by the flap with a large pedicle and skin graft of the donor area secondarily. Minimal and manageable complications were noticed. Discussion: Anatomical reminder and the details of our technique, it is a reliable means for covering defects in the instep and ankle, a comparison was made with other plastic surgery procedures. Advantages and disadvantages of our technique; all stressing the need to take a pedicle wide more than three centimeters. Conclusion: A technique of choice for covering losses of substance in the ankle and the instep. The aim of this study was to report our experience and to assess the reliability of this flap by taking a large pedicle.

Keywords: Defects, ankle, flap, sural, reliability.

Copyright @ 2020: This is an open-access article distributed under the terms of the Creative Commons Attribution license which permits unrestricted use, distribution, and reproduction in any medium for non-commercial use (NonCommercial, or CC-BY-NC) provided the original author and source are credited.

\section{INTRODUCTION}

Reconstructing skin defects of the ankle and foot is really challenging for the plastic surgeon; the lack of locally available tissue for transposition, the lack of a thick fat pad, the proximity to the bone and ligaments, and the poor cutaneous vascularization of these areas all limit the possibilities of a local cutaneous plastic surgeries [1,2].

The role of covering these defects of the ankle is to restore the skin stability and mobility while on standing position and to resist to the shearing forces and traumas that can occur by wearing shoes. In addition, restoring a good sensitivity is also to be taken into account [3].

Depending on their location, extension and the underlying pathologies of the patient, different surgical solutions can be applied (local, regional or free flaps) [4].
Some of these flaps have been abandoned in favor of more reliable and better aesthetic ones. Others are easier to perform with an acceptable result.

The neuro-cutaneous sural flap with distal pedicle is classically performed in covering skin defects of the distal part of the lower limbs. The largest this flap is, the more reliable it can be [5].

We report a series of patients from our department, who benefited from the sural neurocutaneous flap with distal pedicle to cover posttraumatic skin defects of the ankle, using a $3 \mathrm{~cm}$ large fascio-fatty strip containing the pedicle.

The aim of this work is share our experience and to assess the reliability of this technique. 


\section{Material ANd Methods}

This is a retrospective study of a series of 18 patients, from the Plastic surgery department of Mohammed Vth Military Hospital of Rabat, who were treated for ankle skin defects using the sural neurocutaneous flap, from January 2015 to December 2018.

Inclusion criteria : Patients with post-traumatic skin defects of the malleoli, and the upper and medial part of the dorsal aspect of the foot.

Were excluded patients presenting with complex bony defects, skin defects after tumor resection and those interesting the heel.

The analyzed data were age, gender, past medical history (diabetes, or any previous surgeries), the mechanism of the defect, and its location and extension. The follow-up data and the outcome in the short, mid- and long term were also analyzed to detect possible complications.

Both the functional and aesthetic results were evaluated by the surgeon and the patients based on the functional and aesthetic satisfaction that was assessed on an evaluation sheet.

\section{RESULTS}

The following results were recorded: There was a clear male predominance (12 male patients Vs 6 females), with a mean age of 41.6 years, ranging from 20 to 56.

The covering procedure was performed for 10 defects of the internal malleolus, 10 defects of the external malleolus, and 3 at the level of the instep. For ten patients, implantable material for treating bony fractures was already at risk of exposure. For the other patients, bare tendons were noticed. Two of the patients were diabetic, and were managed with oral treatment. Three patients were moderate smokers.

As per our inclusion criteria, all skin defects were post-traumatic, mainly from traffic accidents: six motorcycle accident, 4 pedestrians, 5 car accidents and 3 truck accidents. All patients were scheduled for surgery under spinal anesthesia.

10 out of 18 patients were operated in two stages:

Skin debridement and cleaning of the defect before covering with the sural neuro-cutaneous flap. Skin grafting with a thin dermo-epidermal layer, after 15 days to allow and check the good vascularization of the flap.

For 8 patients, and given the small skin defect, a direct healing was preferred. The outcome for 12 patients was favorable, and allowed for walking two weeks after the surgery while preserving the flap sensitivity.

Six patients encountered post-operative complications: Partial distal necrosis of the flap was noted in 4 patients (2 diabetic, and 2 smokers), requiring debridement under local anesthesia with further favorable outcome. The other two cases experienced local septic complications, that were dealt with adapted antibiotics and local care (based on chlorhexidine type antiseptics), with also a favorable final outcome. All patients had difficulties wearing regular shoes, and were oriented toward adapted shoes.

On the long term follow up, complaints consisted of residual and paroxysmal pain, especially in cold and hot seasons, and excessive walking. These complaints responded well to usual pain killers. Three patients required a degreasing and retouching procedures of the flap.

Overall, the functional and aesthetic result of the reconstruction and the donner site were deemed satisfactory by both the patient and the surgeon.

\section{DISCUSSION}

The sural neuro-cutaneous flap with distal pedicle is a reliable, and easily performed technique for covering almost all skin defects of the lower third of the leg, foot and ankle [6-10].

Thanks to its reliable vascular supply, it can be used for treating chronic ulcers consecutive to trophic disorders, but also for covering skin defects over implantable material, with or without bony infection, provided that the skin debridement is done drastically $[7,11]$.

Other covering techniques to the lower third of the leg hold many disadvantages, listed herein:

- Directed healing: requires a well vascularized underground

- Skin grafting: also requires a well vascularized underground

- Skin expansion prothesis: holds high complication rates

- Random skin flaps and axial skin flaps: high risk of complications due to the low vascular

- supply of the region.

- Free flaps: is a possible option, but better reserved for large and complex skin defects of this

- $\quad$ area. In our department, we usually use the anterolateral thigh flap.

- Artificial skin: should be performed on a non-septic environment, and is expansive.

- Muscular pedicled flaps to the leg:

Soleus flap with lower pedicle: can be applied to cover the lower third of the tibial crest, the internal malleolus, and the distal part of the Achilles tendon. It's 
application is delicate and it is less reliable than its counterpart with upper pedicle. Besides, it is best applied to young patients as it requires very good vascular supply.

Hetero-hamstring flap: the difficulty of this technique lies in the need of a complete immobilization during the autonomation phase. It requires two operating times.

Perforating flap: still can be indicated; needs a doppler workup to analyze underlying vascular possibilities. Regularly performed in our department.

Fascio-cutaneous flap with distal pedicle and the postero-external flap: require two operating times. Distal external supra-malleolar flap: interesting but short.
Anatomy

The medial sural cutaneous nerve arises from the tibial nerve at the lower end of the popliteal fossa and then descends between the two heads of the gastrocnemius muscle. At the lower end of the muscle, it crosses the fascia at the same time as the small saphenous vein. It merges with the fibular communicating branch to become the sural nerve.

The arterial vascularization of the flap comes from the fibular artery, the perforating branches of which join the subcutaneous and fascial arterial network. The most distal and exploitable perforator for the survival of the flap is located approximately 5 to 10 $\mathrm{cm}$ from the tip of the lateral malleolus and will constitute the pivot point of the flap.

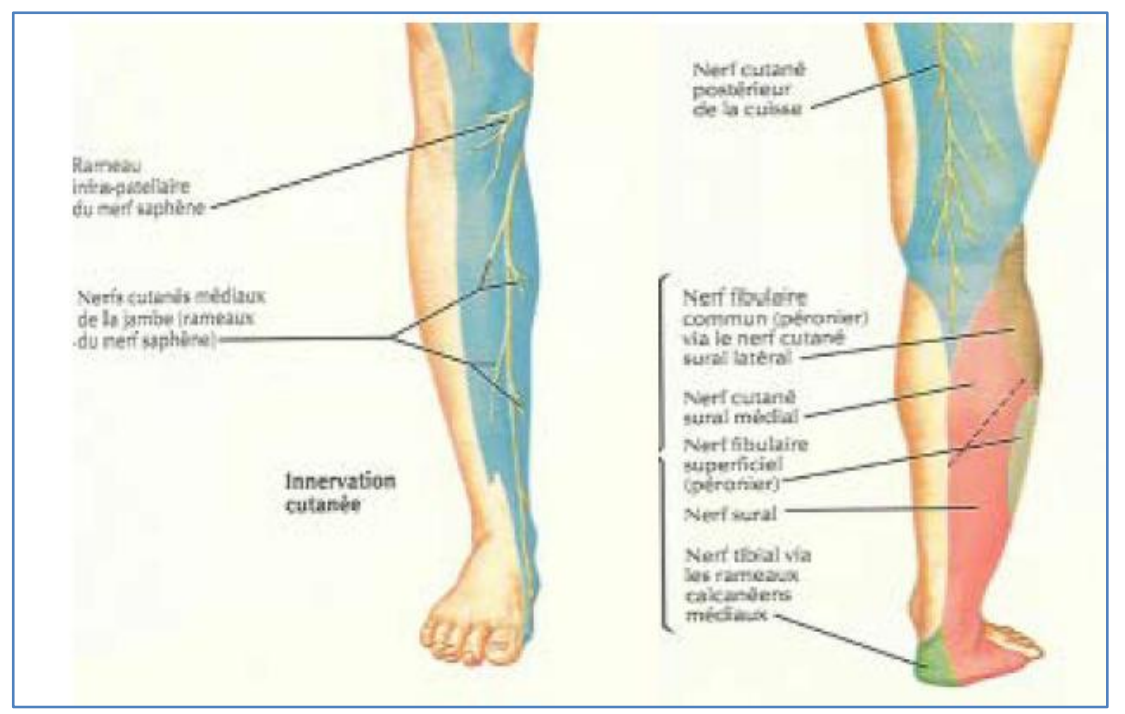

Fig-1: 13 Anatomy of the sural nerve

\section{Lifting technique}

The flap is traced along an axis connecting the junction of the two heads of the gastrognemian muscles to the lateral retro-malleolar groove (corresponding to the path of the sural nerve).

The distal pivot point is placed $7-8 \mathrm{~cm}$ above the relief of the external malleolus to maintain vascular safety of the flap. The pivot point is therefore proximal to the emergence of the distal perforator of the fibular artery. The size of the flap pedicle depends on the skin palette to be taken, but it was $6 \mathrm{~cm}$ on average in the patients we operated on.

An incision between the skin palette and the pivot point is made to allow exposure of the cellulosic space of the flap pedicle. On each side of this incision, we raise two cutaneous-fatty flaps of equal width We incise the edges of the pallet which takes away the fascia, that is sutured with the pallet to avoid the phenomenon of "soaping" which can be origin of skin devascularization of the flap. We then ligate the saphenous vein and cut the sural nerve. By transparency, through the fascia, the nerve and the vein are easily recognizable. This location will guide the removal of the pedicle. The flap is then lifted from proximal to distal, taking over a width of more than three centimeters (our patients) the fascia, the nerve, the artery, the vein and the adjacent hypodermic tissue. (Fig 2; $a$ and $b$ )

We then perform the rotation of the flap and the placement of the skin palette (Fig. 3). The pedicle is exteriorized in the case of our patients, given the poor quality of the surrounding skin of the traumatic defect. (Fig.4). The pedicle can sometimes be carefully tunneled.

Depending on the skin laxity: closure of the donor area at the level of the calf or leave it in directed scarring. To decrease its size, we carry out a purse with non-absorbable thread. No support on the externalized pedicle for 21 days. 
The limb is immobilized $15-21$ days depending on the patient, the flap is autonomous at 21 days.

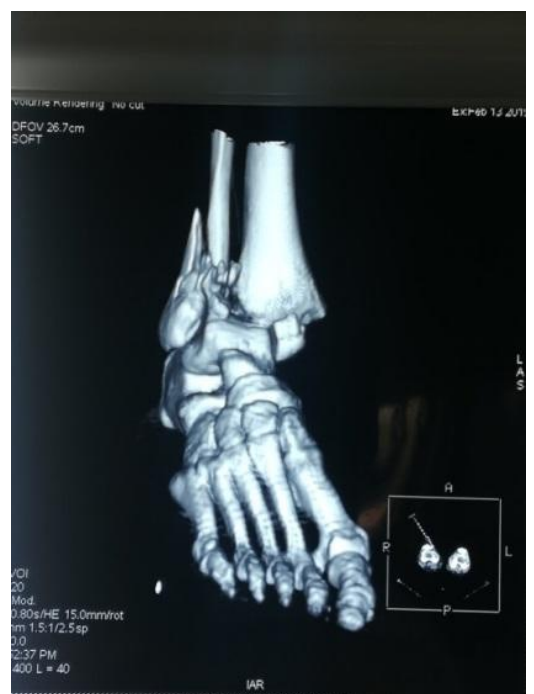

53-year-old man with bimalleolar open fracture following a fall from a ladder

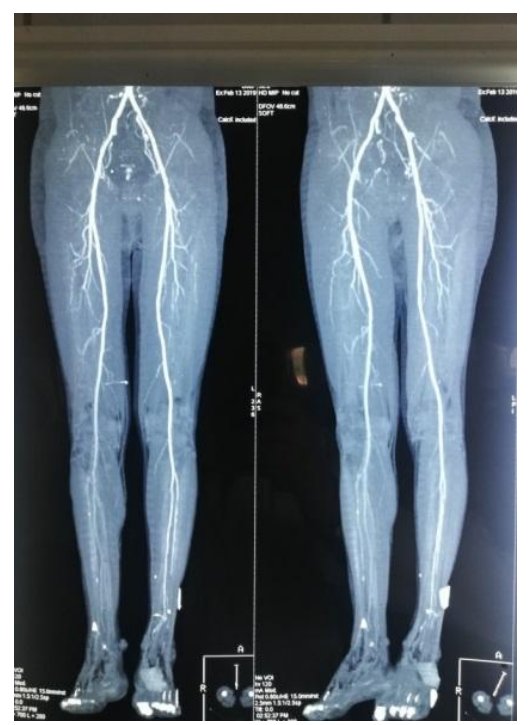

Normal angioscan, suspicion of vascular injury.

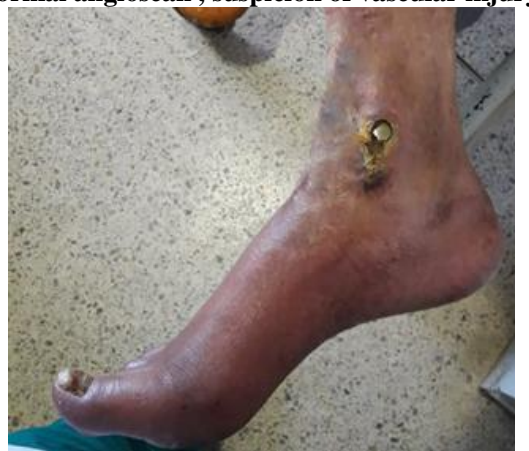

Exposure of the osteosynthesis material, and defect of the internal malleolus

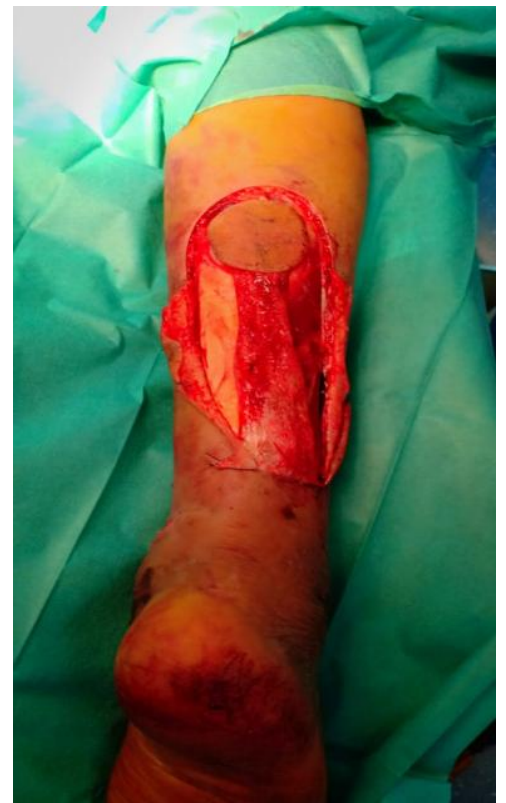

Lifting of the sural neurocutaneous flap with distal pedicle, broad vessel carrier

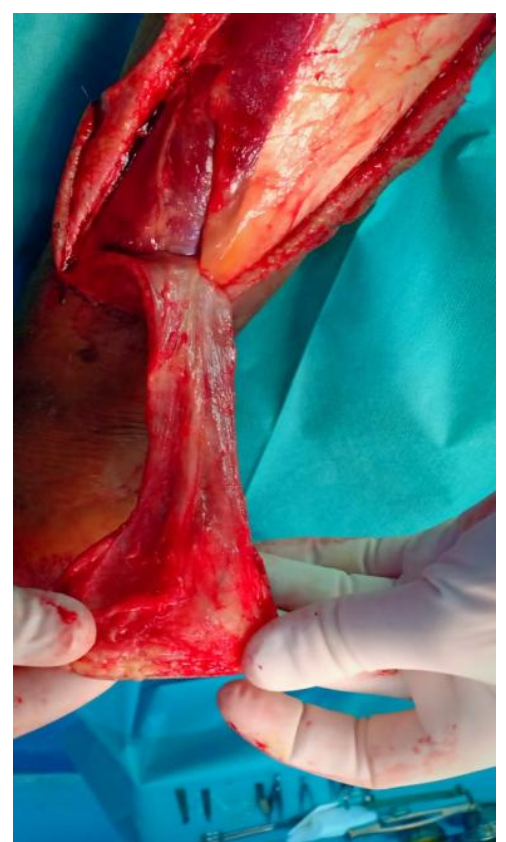

Lifting the flap; visualization of the pedicle 


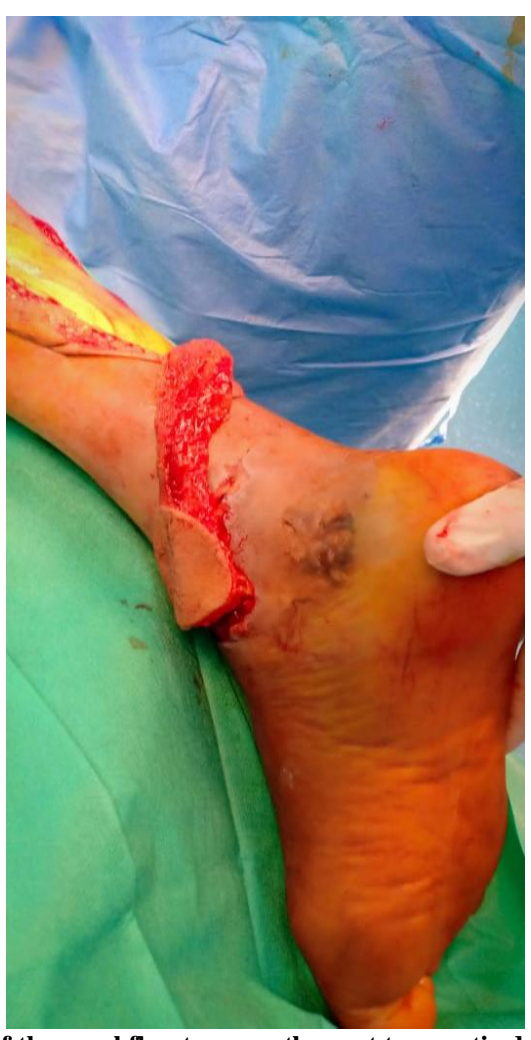

Rotation of the sural flap to cover the post traumatic defect at the level of the internal malleolus, externalized pedicle

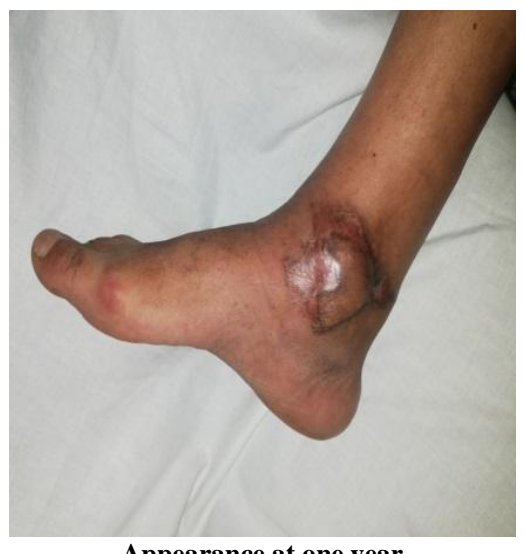

Appearance at one year

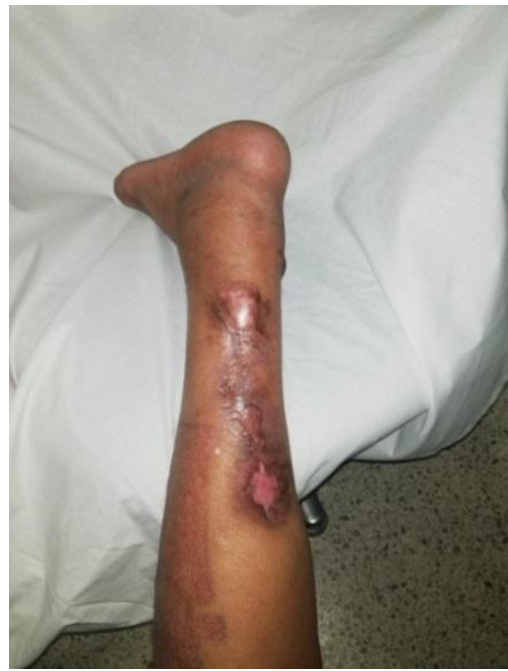

One year, flap donor area and skin grafts

\section{The advantages of the technique}

- Constant anatomical landmarks of the neurovascular axis; technically simple flap; our technique allows the removal of a large pedicle of more than $3 \mathrm{~cm}$ allowing for better venous drainage and consequently less risk of flap necrosis [9].

- No functional sequelae because of no muscle sacrifice.

- The large Arc of rotation.

- No sacrifice of a main vascular axis.

\section{The inconvenients}

- Venous drainage on the contrary: markedly improved by our technical modification; the distal venous anastomosis is no longer necessary.

- Aesthetic sequelae if the donor site receives a transplant.

\section{CONCLUSION}

The neurocutaneous flap with sural pedicle is a technique of choice for covering skin defects with bare noble elements or osteosynthesis material of the ankle and foot. It is simple to perform, without vascular sacrifice, without major sequelae, and finally with low scar ransom.

The purpose of this study was to report our experience and assess the reliability of this flap by removing a wide fascio-fatty strip (of more than $3 \mathrm{~cm}$ ) containing the pedicle, which considerably reduces the risk of necrosis and avoiding performing a distal venous anastomosis to improve venous return which can be the major concern with this type of flap.

\section{REFERENCES}

1. Li X, Cui J, Maharjan S, Lu L, Gong X. Reconstruction of the foot and ankle using pedicled or free flaps: perioperative flap survival analysis. PloS one. 2016 Dec 8;11(12):e0167827.

2. El-Shazly M, Yassin O, Kamal A, Makboul M, Gherardini G. Soft tissue defects of the heel: a surgical reconstruction algorithm based on a retrospective cohort study. J Foot Ankle Surg. 47:145-152, 2008.

3. Shen ZL, Jia WX, Hou MZ, Huang XQ, Cai YX, Wang L, Huang YX. Application of flaps for coverage of foot wounds with tendon and bone exposure based on a subunit principle. InInnovations in Plastic and Aesthetic Surgery). Springer, Berlin, Heidelberg. 2008: 119-126

4. Revol S, Servant JM, Bauzet P. Les possibilités actuelles de couverture des pieds cutanée posttraumatique des jambes et des talons. J Trauma. 1981; 2: 149-56.

5. Chesnier I, Bali D, Casanova D, Legré R, Magalon G. Flaps in lower limb reconstruction: a 10-year retrospective review of 157 pedicled flaps. Ann Chir Plast Esthet. 2012;57:328-35. 
6. Hasegawa M, Torri S, Katoh H, Esaki S. The distally based superficial artery flap. Plast Reconstrut Surg. 1994; 93: 2012.

7. Spyriounis PK. The use of the distally based neuroveinocutaneous sural flap. Rom J Plast Surg. 2000;8:261

8. Touam C, Rostoucher P, Bhatia A, Oberlin C. Comparative study of two series of distally based fasciocutaneous flaps for coverage of the lower one-fourth of the leg, the ankle, and the foot. Plastic and reconstructive surgery. 2001 Feb;107(2):383-92.

9. Rajacic N, Darweesh M, Jayakrishnan K, Gang RK and Kojic S. The distally based superficial sural flap for reconstruction of the lower leg and foot
10. Tu, Yuan-Kun MD; Ueng, Steve Wen-Neng MD; Yeh, Wen-Lin MD; Wang, Kun-Chuang MD Reconstruction of Ankle and Heel Defects by a Modified Wide-Base Reverse Sural Flap

11. Tu YK, Ueng SWN, Yeh WL, Wang KC. Reconstruction of ankle and heel defects by à modified wide-base reverse sural flap. J Trauma. 1999;47:82.

12. Townsend PL. An inferiorly based Soleus muscle flap. Br. J. Plast. Surg. 1978; 31: 210-213

13. Frank-H Netter; Atlas d'anatomie humaine.

14. Tanakia Y, Tajima S. The influence of arterial inflow and venous outflow on the survival of reversed-flow island flaps: an experimental study. Plast Reconstr Surg. 1997;99:2021. 